\title{
Outcomes in patients with nonerosive reflux disease treated with a proton pump inhibitor and alginic acid \pm glycyrrhetinic acid and anthocyanosides
}

\author{
This article was published in the following Dove Press journal: \\ Clinical and Experimental Gastroenterology \\ 26 March 2013 \\ Number of times this article has been viewed
}

\author{
Francesco Di Pierro' \\ Mario Gatti ${ }^{2}$ \\ Giuliana Rapacioli ${ }^{3}$ \\ Leandro Ivaldi ${ }^{4}$ \\ 'Velleja Research, Milan, \\ ${ }^{2}$ Gastroenterology Department, \\ Giussano Hospital, Monza-Brianza, \\ ${ }^{3}$ AIOR, Piacenza, ${ }^{4}$ Digestive \\ Endoscopic Department, Ceva \\ Hospital, Ceva, Cuneo, Italy
}

\begin{abstract}
Background: The purpose of this study was to compare the efficacy of alginic acid alone versus alginic acid combined with low doses of pure glycyrrhetinic acid and bilberry anthocyanosides as an addon to conventional proton pump inhibitor therapy in relieving symptoms associated with nonerosive reflux disease.
\end{abstract}

Methods: This prospective, randomized, 8-week, open-label trial was conducted at two centers. Sixty-three patients with persistent symptoms of gastroesophageal reflux disease and normal upper gastrointestinal endoscopy were eligible for the study. Patients in group A ( $n=31)$ were treated with pantoprazole and a formula $\left(\right.$ Mirgeal $^{\circledR}$ ) containing alginic acid and low doses of pure glycyrrhetinic acid + standardized Vaccinium myrtillus extract for 4 weeks, then crossed over to the multi-ingredient formula for a further 4 weeks. Patients in group B $(n=32)$ were treated pantoprazole and alginic acid alone twice daily, then crossed over to alginic acid twice daily for a further 4 weeks. Efficacy was assessed by medical evaluation of a symptom relief score, estimated using a visual analog scale (0-10). Side effects, tolerability, and compliance were also assessed.

Results: Of the 63 patients enrolled in the study, 58 (29 in group A and 29 in group B) completed the 8 -week trial. The baseline characteristics were comparable between the two groups. During the study, significant differences were recorded in symptom scores for both groups. In group A, symptoms of chest pain, heartburn, and abdominal swelling were less serious than in group B. Treatment A was better tolerated, did not induce hypertension, and had fewer side effects than treatment B. No significant differences in compliance were found between the two groups.

Conclusion: Use of low doses of pure glycyrrhetinic acid + bilberry anthocyanosides, together with alginic acid as addon therapy, substantially improves symptoms in patients with nonerosive reflux disease without increasing side effects or worsening tolerability or compliance.

Keywords: proton pump inhibitors, alginic acid, glycyrrhetinic acid, anthocyanosides, nonerosive reflux disease, gastroesophageal reflux disease

\section{Introduction}

Gastroesophageal reflux disease (GERD) is a chronic relapsing medical condition that involves reflux of gastric contents into the esophagus, and causes a multitude of unpleasant symptoms, including heartburn, sore throat, chest pain, abdominal swelling, cough, and regurgitation. GERD has been demonstrated to have a significant negative impact on quality of life in affected patients and may even impair their daily activities. ${ }^{1}$ The prevalence of GERD has markedly increased over the last two decades,
Correspondence: Francesco Di Pierro Velleja Research, Viale Lunigiana 23.

20I25, Milan, Italy

Tel +393495527663

Fax +390523511894

Email f.dipierro@vellejaresearch.com 
and affects $20 \%-40 \%$ of the Western population. ${ }^{2-5}$ This prevalence is expected to rise further with time. ${ }^{6}$ Nonerosive reflux disease (NERD) has been defined by the Vevey NERD consensus group as a subcategory of GERD that is characterized by reflux-related symptoms, with an absence of esophageal mucosal erosions or breaks at conventional endoscopy. ${ }^{7}$ About two-thirds of patients with typical GERD symptoms, such as heartburn, belching, cough, nausea, sore throat, and voice changes, do not exhibit any erosive changes on upper gastrointestinal endoscopic assessment. ${ }^{2}$ The complex pathophysiology of NERD and the exact mechanisms by which the associated symptoms are caused remain unclear. ${ }^{7}$ However, it is evident that the degree of acidity and duration of esophageal exposure to acid play a role in the symptomatology of NERD. These factors are not different from the precipitants of moderate erosive reflux disease..$^{7-11}$

The majority of patients with GERD use acid-suppressing medications to control their symptoms. Unlike moderate erosive reflux disease, the symptoms of NERD are more difficult to control and tend to have a lower response rate to treatment, even to the most potent proton pump inhibitors. ${ }^{12,13}$ Nonetheless, initial management of NERD is similar to that of GERD, and includes use of a proton pump inhibitor or H2receptor antagonist, along with alginic acid-based products. Consequently, we attempted to verify whether it was possible to ameliorate the initial management of NERD clinically by also exploiting, for instance, the role played by alginic acid as a drug delivery system. Standardized Glycyrrhiza glabra (common licorice) extract is a well known sweetener, and is also endowed with anti-inflammatory, cytoprotective, and antiulcer properties. ${ }^{14}$ The "sweetener" activity is strictly related to the presence of a molecule known as glycyrrhizic acid, hydrolysis of which releases 18-beta-glycyrrhetinic acid as a result of detachment of a sugar moiety. The latter is responsible for both the anti-inflammatory, cytoprotective, and antiulcer activity of this compound. In fact, licorice-based products devoid of glycyrrhetinic acid or its prodrug are also devoid of any biological activity. ${ }^{15}$ The antiulcer effect of licorice-based products is observed with doses of glycyrrhetinic acid around 400-500 mg/day, and with doses of its prodrug ranging from $750 \mathrm{mg} /$ day to $1000 \mathrm{mg} /$ day. ${ }^{16,17} \mathrm{In}$ any case, in most people, these doses induce an increase in blood pressure, loss of potassium, sodium retention, and weight gain. These side effects already occur following consumption of $100 \mathrm{mg}$ glycyrrhizic acid (the glycyrrhetinic acid prodrug), corresponding to $50 \mathrm{mg}$ of pure glycyrrhetinic acid, a dose that is about 8-10 times lower than that required to obtain a clear cytoprotective and antiulcer effect. ${ }^{18}$ Increased blood pressure, potassium loss, and weight gain are all side effects arising from sodium retention induced by glycyrrhetinic acid. Once glycyrrhetinic acid has been absorbed, and in particular following liver glucuronidation, it strongly inhibits the kidney isoform of 11-beta hydroxysteroid dehydrogenase. This enzyme induces overstimulation of the mineralocorticoid receptor because of nonmetabolized cortisol, which in turn results in sodium-dependent hypertension, with weight gain and loss of potassium. ${ }^{19}$

On the other hand, the mechanism by which glycyrrhetinic acid exerts its cytoprotective and antiulcer activity is quite different. Once glycyrrhetinic acid reaches the circulation, it spreads into the different organs, including into gastric tissue, where it inhibits an enzyme known as $15-\mathrm{OH}$ prostaglandin dehydrogenase, which is normally involved in inactivating two prostaglandins endowed with strong cytoprotective activity, ie, prostaglandins E2 and F2 $\alpha$. Glycyrrhetinic acid-containing licorice derivatives have beneficial effects on the gastric mucosa because they prolong the presence of these prostaglandins. ${ }^{20}$ The longer glycyrrhetinic acid remains in direct contact with the gastric epithelium, the more it can play a cytoprotective role. The time required by the bolus to transit an empty stomach is about 35-40 minutes, and this time is too short to ensure a good cytoprotective effect, especially if using very low doses of the active ingredient, glycyrrhetinic acid. On a full stomach, the bolus transit time may be longer, but the presence of food would limit direct contact between glycyrrhetinic acid and the epithelium of the stomach.

Therefore, in order to exploit the benefits of glycyrrhetinic acid to the gastric mucosa without risking serious side effects, we decided to use alginic acid as a drug delivery system for low doses of glycyrrhetinic acid which, entrapped inside the alginate, is released in about 2-3 hours, according to the alginate dissolution time in the stomach, allowing longlasting direct contact between glycyrrhetinic acid and the gastric mucosa. Use of low doses of glycyrrhetinic acid, below the minimum dose known to induce side effects, seems to reduce the risk of consequences of sodium retention. In confirmation of earlier reports, ${ }^{21,22}$ some authors have recently reported a strong cytoprotective role at the gastric mucosa played by anthocyanosides, a class of polyphenols extracted from Vaccinium myrtillus (the common bilberry). ${ }^{23}$ On this assumption, we developed an alginate-based cytoprotective product containing pure glycyrrhetinic acid from licorice and anthocyanosides from bilberry. The objective of our study was to compare the efficacy of low doses of glycyrrhetinic acid and anthocyanosides, entrapped in an alginate matrix, as 
an addon therapy to proton pump inhibitors in the treatment of patients with NERD.

\section{Materials and methods}

We conducted a prospective, randomized, 8-week, openlabel two-center study in patients with a diagnosis of NERD. Patients considered eligible for enrollment had to be over 18 years of age, suffer from persistent typical or atypical symptoms of GERD, and have a negative upper gastrointestinal endoscopy. Exclusion criteria were age younger than 18 years or over 75 years, any allergy to established medications, any degree of esophagitis or mucosal damage at upper gastrointestinal endoscopy, pregnancy, or use of a proton pump inhibitor or H2-receptor antagonist in the 2 weeks prior to enrollment in the study. Sixty-three patients who attended the two study medical centers with persistent GERD symptoms and a normal upper gastrointestinal endoscopy were considered candidates for the study. After signing their written informed consent, the patients were asked to complete a questionnaire concerning their personal data. They were then randomized by an independent investigator using a computer-generated random number table to one of two groups: one receiving pantoprazole as well as Mirgeal ${ }^{\circledR}$ (PharmExtracta, Pontenure, Italy, group A) and one receiving pantoprazole along with alginate (group B). Patients in group A were allowed a maximum oral pantoprazole dose of $40 \mathrm{mg}$ (20 mg tablets, twice daily) along with Mirgeal containing the same dose of alginate as that administered in group B (see below), plus $50 \mathrm{mg}$ of pure glycyrrhetinic acid (25 mg, twice daily) $+40 \mathrm{mg}$ of anthocyanosides $(20 \mathrm{mg}$, twice daily); those in group B were allowed the same dosage of pantoprazole as that used in group A, along with $1000 \mathrm{mg}$ alginate (500 mg sachets, twice daily). Twenty-nine of the 31 patients in group A and 29 of the 32 patients in group B completed the study, indicating good adherence. Only these 58 individuals were considered valid for statistical analysis. Seven of the 29 subjects in group A were receiving concomitant therapy that was not considered to have an effect on the study results. These seven patients were being treated with antihypertensives, statins, levothyroxine, ticlopidine, or lowdose aspirin. Nine of the 29 subjects in group B were also on treatment with other drugs, ie, low-dose aspirin, levothyroxine, statins, or antidepressants. During the first 4 weeks of the study, group A subjects was treated daily with pantoprazole and Mirgeal, and then crossed over to Mirgeal alone for a further 4 weeks. Similarly, group B subjects were treated for 4 weeks with pantoprazole and alginate, then crossed over to alginate alone for a further 4 weeks. One of the researchers was responsible for contacting the patients by phone on a daily basis over the 8-week study period to assess the number of pills and sachets taken, the need for rescue medication, and the occurrence of adverse events. Every 15 days until the end of the 8-week study period, patients were asked about their global symptom relief, along with side effects, tolerability, and compliance.

The study was done in a routine clinical practice setting, following international guidelines and in line with the principles outlined in the Declaration of Helsinki. This study was carried out in two Italian outpatient clinics, ie, the Department of Gastroenterology, Giussano Hospital, Monza-Brianza, and the Digestive Endoscopic Department, Ceva Hospital, Ceva, Italy, after informing the local ethical boards. In Italy, pantoprazole and alginate are registered as over-the-counter drugs. Mirgeal, in agreement with Italian law number 169/2004, was notified to the Minister of Health on July 5, 2011 (registration number pending) and registered as a food supplement.

The primary efficacy endpoints of the study were assessed according to relief of subjective global symptoms (chest pain, heartburn, regurgitation, abdominal swelling, hoarseness, cough, throat pain), using an adapted version of the visual analog scale (scale 0-10) developed by Scott and Huskisson. ${ }^{24}$ Secondary endpoints included the occurrence of side effects, tolerability, and compliance. Tolerability and compliance were scored as very poor, poor, fair, good, and excellent.

\section{Statistical analysis}

The statistical analysis used to check the data variations was the between/within-subjects analysis of variance applied to two factors, ie, treatment and period. The Tukey-Kramer method was used for multiple comparative analysis. The Wilcoxon/Kruskal-Wallis method was used to evaluate the global outcome with regard to symptoms.

\section{Results}

The main aim of our trial was to compare the efficacy of low doses of GA and anthocyanosides, entrapped in an alginate matrix, as an add-on therapy to pantoprazole, to treat patients with NERD. To reach our objective, we compared 2 types of therapy. The first - Group A - consisted of pantoprazole along with alginate, which were mixed with 2 other active compounds - GA and anthocyanosides from bilberry fruits - at lower doses than those normally used; the secondGroup B - consisted of pantoprazole along with alginate as is. As shown in Table 1, the personal and baseline characteristic of the 2 groups did not differ statistically. 
Table I Personal and baseline characteristics of subjects $(n=29$ per group)

\begin{tabular}{llll}
\hline Variable & Group A & Group B & P \\
\hline Age (years) & $52.9 \pm 15.2$ & $47.3 \pm 12.6$ & 0.1358 \\
Height $(\mathrm{cm})$ & $164.3 \pm 24.4$ & $167.1 \pm 19.5$ & 0.6317 \\
Weight $(\mathrm{kg})$ & $71.9 \pm 11.0$ & $71.6 \pm 13.8$ & 0.9249 \\
Treatment & $65.7 \pm 9.0$ & $63.1 \pm 4.0$ & 0.1734 \\
duration (days) & & & \\
Males & $\mathrm{II}$ & 15 & 0.2909 \\
Females & 18 & 14 & 0.3167 \\
\hline
\end{tabular}

Note: Results are presented as the mean \pm standard deviation.

With regard to chest pain, as shown in Table 2, Group A treatment exhibited a better reduction in the score value. This had been statistically significant since the second medical check. From the third examination onwards, differences became even more important. Treatment B showed a reduction in the scores until the second medical check. From the third medical check onwards, no further reduction was observable.

With regard to heartburn, as shown in Table 3, Group A treatment exhibited a better reduction in the score value. This had been statistically significant since the second medical check. From the third examination onwards, differences became even more important. Treatment B showed a reduction in the scores until the second medical check. From the third medical check onwards, no further reduction was observable.

With regard to abdominal swelling, as shown in Table 4, Group A treatment shows a better reduction in the score value. This is statistically significant since the second medical check. From the third examination onwards, differences became even more important. Treatment B show a reduction in the scores until the second medical check. From the third medical check onwards, no further reduction was observable.

With regard to regurgitation, as shown in Table 5, Group A treatment exhibited a better reduction in the score value only at the end of the treatment (medical checks 4 and 5) as the curves of the 2 treatments overlapped from the baseline till the 3rd medical check.
With regard to hoarseness, as shown in Table 6, Group A treatment exhibited a better reduction in the score value only at the end of the treatment (medical checks 4 and 5) as the curves of the 2 treatments overlapped from the baseline till the $3 \mathrm{rd}$ medical check. With regard to cough, as shown in Table 7, only at the 5 th medical examination did the 2 treatments show some statistically significant differences. With regard to throat pain, no differences were seen between the 2 treatments even if a slight tendency to improvement was still observed (Table 8).

As shown in Table 9 (pooling of all scores), the evaluation of the global outcome is similar in the 2 groups at baseline. Starting from the 2 nd medical check the clinical action played by the treatment A versus treatment B become very relevant and statistically significant.

As for side effects (data not shown in the tables above), they affected 10 individuals. Two $(6.90 \%)$ belonging to group A, and 8 (27.59\%) belonging to group B. This difference appears significant $(P=0.0370)$ in favor of Group A. It is worth noting that, especially for Group A treated with pure GA (licorice derivative), none of the individuals reported any increase in terms of blood pressure or weight gain (data not shown). With regard to tolerability (Table 10), treatment A seemed to be better tolerated. In terms of compliance, the 2 treatments resulted to be totally overlapping (Table 11).

\section{Discussion}

The aim of our study was to compare the efficacy of low doses of glycyrrhetinic acid and anthocyanosides entrapped in an alginate matrix as an addon to proton pump inhibitor therapy in patients with NERD. To this end, we tested two treatment patterns: in group A we used oral pantoprazole $20 \mathrm{mg}$ tablets twice daily along with Mirgeal ${ }^{\circledR}$ (a multi-ingredient formulation containing alginate $500 \mathrm{mg}+$ pure glycyrrhetinic acid $25 \mathrm{mg}$ and bilberry anthocyanosides $20 \mathrm{mg}$ ) twice daily; in group B we used the same dose of pantoprazole as in group A, along with alginate $500 \mathrm{mg}$ sachets twice daily. At the end of the 8-week study, 29 of the 31 patients enrolled in group A and 29 of the 32 enrolled in group B completed the study, indicating good adherence with therapy.

Table 2 Symptom scores for chest pain

\begin{tabular}{llllll}
\hline Group & $\mathbf{n}$ & Baseline & MC 2 & MC 3 & MC 5 \\
\hline A & 24 & $4.08 \pm 2.50$ & $1.96 \pm 1.88$ & $1.13 \pm 1.33$ & $1.08 \pm 1.50$ \\
B & 22 & $5.06 \pm 2.32$ & $3.82 \pm 2.11$ & $4.23 \pm 1.48$ & $3.64 \pm 1.99$ \\
$P$ value & & NS & $<0.05$ & $<0.05$ & $3.59 \pm 2.24$ \\
\hline
\end{tabular}

Note: Results are presented as the mean \pm standard deviation.

Abbreviations: MC, medical check; NS, not statistically significant. 
Table 3 Symptom scores for heartburn

\begin{tabular}{lllllll}
\hline Group & $\mathbf{n}$ & Baseline & MC 2 & MC 3 & MC 4 & MC 5 \\
\hline A & 24 & $5.83 \pm 1.99$ & $1.96 \pm 1.68$ & $1.21 \pm 1.10$ & $0.96 \pm 1.00$ & $1.50 \pm 1.32$ \\
B & 27 & $5.93 \pm 2.38$ & $3.41 \pm 2.56$ & $3.26 \pm 2.16$ & $3.41 \pm 1.95$ & $3.19 \pm 2.09$ \\
$P$ value & & NS & $<0.05$ & $<0.05$ & $<0.05$ & $<0.05$ \\
\hline
\end{tabular}

Note: Results are presented as the mean \pm standard deviation.

Abbreviations: MC, medical check; NS, not statistically significant.

Table 4 Symptom scores for abdominal swelling

\begin{tabular}{lllllll}
\hline Group & $\mathbf{n}$ & Baseline & MC 2 & MC 3 & MC 4 & MC 5 \\
\hline A & 24 & $5.75 \pm 2.57$ & $3.08 \pm 2.26$ & $2.21 \pm 1.96$ & $2.25 \pm 1.54$ & $2.21 \pm 1.74$ \\
B & 24 & $6.71 \pm 2.22$ & $5.21 \pm 2.19$ & $5.33 \pm 1.88$ & $5.04 \pm 1.68$ & $4.54 \pm 1.91$ \\
$P$ value & & NS & $<0.05$ & $<0.05$ & $<0.05$ & $<0.05$ \\
\hline
\end{tabular}

Note: Results are presented as the mean \pm standard deviation.

Abbreviations: MC, medical check; NS, not statistically significant.

Table 5 Symptom scores for regurgitation

\begin{tabular}{lllllll}
\hline Group & $\mathbf{n}$ & Baseline & MC 2 & MC 3 & MC 4 & MC 5 \\
\hline A & 15 & $5.67 \pm 2.26$ & $2.00 \pm 1.77$ & $1.20 \pm 1.42$ & $0.80 \pm 1.15$ & $0.60 \pm 0.91$ \\
B & 19 & $4.00 \pm 2.89$ & $1.16 \pm 1.71$ & $1.95 \pm 2.46$ & $2.95 \pm 2.76$ & $2.89 \pm 2.85$ \\
$P$ value & & NS & NS & NS & $<0.05$ & $<0.05$ \\
\hline
\end{tabular}

Note: Results are presented as the mean \pm standard deviation.

Abbreviations: MC, medical check; NS, not statistically significant.

Table 6 Symptom scores for hoarseness

\begin{tabular}{lllllll}
\hline Group & $\mathbf{n}$ & Baseline & MC 2 & MC 3 & MC 4 & MC 5 \\
\hline A & II & $4.27 \pm 2.41$ & $1.91 \pm 1.76$ & $1.45 \pm 1.51$ & $1.09 \pm 1.30$ & $0.91 \pm 1.14$ \\
B & I4 & $4.50 \pm 2.85$ & $2.57 \pm 3.01$ & $2.57 \pm 2.44$ & $3.00 \pm 2.83$ & $3.21 \pm 2.86$ \\
$P$ value & & NS & NS & NS & $<0.05$ & $<0.05$ \\
\hline
\end{tabular}

Note: Results are presented as the mean \pm standard deviation.

Abbreviations: MC, medical check; NS, not statistically significant.

Table 7 Symptom scores for cough

\begin{tabular}{lllllll}
\hline Group & $\mathbf{n}$ & Baseline & MC 2 & MC 3 & MC 4 & MC 5 \\
\hline A & 15 & $2.80 \pm 2.34$ & $1.13 \pm 1.77$ & $0.93 \pm 1.36$ & $1.13 \pm 1.64$ & $0.47 \pm 1.13$ \\
B & 17 & $2.76 \pm 2.99$ & $1.59 \pm 2.27$ & $1.65 \pm 2.15$ & $1.94 \pm 2.54$ & $2.00 \pm 2.85$ \\
$P$ value & & NS & NS & NS & NS & $<0.05$ \\
\hline
\end{tabular}

Note: Results are presented as the mean \pm standard deviation.

Abbreviations: MC, medical check; NS, not statistically significant.

Table 8 Symptom scores for throat pain

\begin{tabular}{lllllll}
\hline Group & $\mathbf{n}$ & Baseline & MC 2 & MC 3 & MC 4 & MC 5 \\
\hline A & 14 & $4.00 \pm 3.09$ & $1.43 \pm 1.50$ & $0.64 \pm 0.93$ & $1.14 \pm 1.66$ & $0.36 \pm 0.63$ \\
B & 14 & $3.71 \pm 2.20$ & $1.79 \pm 2.22$ & $1.57 \pm 1.87$ & $1.79 \pm 2.19$ & $2.07 \pm 2.09$ \\
$P$ value & & NS & NS & NS & NS & NS \\
\hline
\end{tabular}

Note: Results are presented as the mean \pm standard deviation.

Abbreviations: MC, medical check; NS, not statistically significant. 
Table 9 Mean global outcome

\begin{tabular}{llllll}
\hline Group & Baseline & MC 2 & MC 3 & MC 4 & MC 5 \\
\hline A & 20.9 & 8.8 & 5.5 & 5.5 & 5.0 \\
B & 23.7 & 14.2 & 14.9 & $\mathrm{I} 5.4$ & 15.1 \\
Wilcoxon S & 974.5 & 1063.5 & 1206.5 & $121 \mathrm{I} .5$ & $120 \mathrm{I} .0$ \\
$P$ value & 0.0637 & $0.00 \mathrm{I} 2$ & $<0.000 \mathrm{I}$ & $<0.000 \mathrm{I}$ & $<0.000 \mathrm{I}$ \\
\hline
\end{tabular}

Note: Results are presented as the mean \pm standard deviation. Abbreviation: MC, medical check.

Analysis of our data show that both therapies were effective in ameliorating symptoms. However, comparison of the two treatment options showed that Mirgeal ${ }^{\circledR}$ as an addon to proton pump inhibitor therapy in patients affected by NERD was more effective than use of alginate alone in controlling both typical and atypical symptoms. In particular, the use of the multi-ingredient formula, rather than alginate alone, resulted in a statistically significant reduction in chest pain, heartburn, and abdominal pain from the second medical check onwards. Regurgitation and hoarseness was found to be improved at the fourth medical check, and cough was found to have improved significantly only at the fifth medical check, while no statistically significant changes were observed in throat pain.

Even when the statistical analysis did not show any major difference, treatment with glycyrrhetinic acid + anthocyanosides added to alginate achieved a slight improvement versus use of alginate alone. Mirgeal also showed advantages in terms of side effects (two subjects of 29 versus eight of 29) and tolerability. It is of note that use of glycyrrhetinic acid at a $25 \mathrm{mg}$ dose (which is 8-10 times lower than the dose reported to determine cytoprotective and antiulcer activity) did not result in any increase in blood pressure or weight gain.

In conclusion, this trial seems to suggest some important data. First, glycyrrhetinic acid (or even licorice extract containing glycyrrhetinic acid or the glycyrrhetinic acid prodrug) can enhance the results obtained using proton pump inhibitor therapy without risking hypertension simply by using very low doses in a pharmaceutical formulation (alginate matrix) that allows the active ingredient to remain in direct contact with the gastric mucosa for 2-3 hours. Such low doses are required to avoid the sodium retention problem with the consequences of

Table 10 Tolerability outcome

\begin{tabular}{llll}
\hline Tolerability & Group A (n) & Group B (n) & $\mathbf{P}$ \\
\hline Poor & 0 & 2 & NS \\
Fair & 7 & 10 & NS \\
Good & 11 & 17 & NS \\
Excellent & 11 & 0 & 0.002 \\
\hline
\end{tabular}

Abbreviation: NS, not statistically significant.
Table I I Results for compliance

\begin{tabular}{llll}
\hline Compliance & Group A (n) & Group B (n) & $\boldsymbol{P}$ \\
\hline Fair & 3 & I & NS \\
Good & 17 & 19 & NS \\
Excellent & 9 & 9 & NS \\
\hline Abbreviation: NS, not statistically significant. & &
\end{tabular}

increased blood pressure and weight gain. Second, consistent with recent papers describing the cytoprotective role played by anthocyanosides, this trial seems to confirm the results obtained in animal studies since the 1980s.

Our pilot study has some limitations, in particular its open-label nature. Although patients were randomized to different arms, they were aware of the treatment arm they had been allocated to. This may have created some bias, especially if these patients had been previously treated with products similar to those they were assigned to. Further, given the lack of dose-response observed in many proton pump inhibitor studies treating patients with NERD, the results of the current study appear quite remarkable, particularly if we consider that all patients were already treated with both a proton pump inhibitor twice daily and alginic acid twice daily. Moreover, from a scientific standpoint, the study does not clarify if the benefits are mainly due to the presence of glycyrrhetinic acid or to bilberry anthocyanosides, or both. An experimental rat model of mild gastric ulcers induced by low doses of indomethacin is under way in an attempt to answer this question. The study sample size was also relatively small, and further investigations in a larger number of patients are necessary to corroborate our data. Finally, our study duration was short and did not evaluate a possible on-demand approach in terms of use of pantoprazole during the second 4 weeks of the study, where the only treatment possible was alginate with or without glycyrrhetinic acid + anthocyanosides.

\section{Disclosure}

FDP is the main formulator of Mirgeal. The remaining authors report no conflicts of interest in this work.

\section{References}

1. Carlsson R, Dent J, Watts R, et al. Gastro-oesophageal reflux disease in primary care: an international study of different treatment strategies with omeprazole. International GORD Study Group. Eur J Gastroenterol Hepatol. 1998;10:119-124.

2. Fass R, Fennerty MB, Vakil N. Nonerosive reflux disease - current concepts and dilemmas. Am J Gastroenterol. 2001;96:303-314.

3. Locke GR, Talley NJ, Fett SL, Zinsmeister AR, Melton LJ. Prevalence and clinical spectrum of gastroesophageal reflux: a population-based study in Olmsted County, Minnesota. Gastroenterology. 1997;112: $1448-1456$. 
4. Ronkainen J, Aro P, Storskrubb T, et al. High prevalence of gastroesophageal reflux symptoms and esophagitis with or without symptoms in the general adult Swedish population: a Kalixanda study report. Scand J Gastroenterol. 2005;40:275-285.

5. Mishima I, Adachi K, Arima N, et al. Prevalence of endoscopically negative and positive gastroesophageal reflux disease in the Japanese. Scand J Gastroenterol. 2005;40:1005-1009.

6. El-Serag HB. Time trends of gastroesophageal reflux disease: a systematic review. Clin Gastroenterol Hepatol. 2007;5:17-26.

7. Modlin IM, Hunt RH, Malfertheiner P, et al. Diagnosis and management of non-erosive reflux disease - the Vevey NERD consensus group. Digestion. 2009;80:74-88.

8. Winter JW, Heading RC. The nonerosive reflux diseasegastroesophageal reflux disease controversy. Curr Opin Gastroenterol. 2008;24:509-515.

9. Martínek J, Benes M, Hucl T, Drastich P, Stirand P, Spicák J. Non-erosive and erosive gastroesophageal reflux diseases: no difference with regard to reflux pattern and motility abnormalities. Scand J Gastroenterol. 2008;43:794-800.

10. Shapiro M, Green C, Faybush EM, Esquivel RF, Fass R. The extent of oesophageal acid exposure overlap among the different gastro-esophageal reflux disease groups. Aliment Pharmacol Ther. 2006;23: 321-329.

11. Martinez SD, Malagon IB, Garewal HS, Cui H, Fass R. Non-erosive reflux disease (NERD) - acid reflux and symptom patterns. Aliment Pharmacol Ther. 2003;17:537-545.

12. Dean BB, Gano AD, Knight K, Ofman JJ, Fass R. Effectiveness of proton pump inhibitors in nonerosive reflux disease. Clin Gastroenterol Hepatol. 2004;2:656-664.

13. Galmiche JP. Non-erosive reflux disease and atypical gastro-esophageal reflux disease manifestations: treatment results. Drugs. 2006;66 Suppl $1: 7-13$.
14. Asl MN, Hosseinzadeh H. Review of pharmacological effects of Glycyrrhiza sp. and its bioactive compounds. Phytother Res. 2008;22(6):709-724.

15. Engqvist A, von Feilitzen F, Pyk E, Reichard H. Double-blind trial of deglycyrrhizinated liquorice in gastric ulcer. Gut. 1973;14:711-715.

16. Ferrari P, Sansonnens A, Dick B, Frey FJ. In vivo 11beta-HSD-2 activity: variability, salt-sensitivity, and effect of licorice. Hypertension. 2001;38:1330-1336.

17. Serra A, Uehlinger DE, Ferrari P, et al. Glycyrrhetinic acid decreases plasma potassium concentrations in patients with anuria. J Am Soc Nephrol. 2002;13:191-196.

18. Størmer FC, Reistad R, Alexander J. Glycyrrhizic acid in liquorice: evaluation of health hazard. Food Chem Toxicol. 1993;31:303-312.

19. Kato H, Kanaoka M, Yano S, Kobayashi M. 3-Monoglucuronylglycyrrhetinic acid is a major metabolite that causes licoriceinduced pseudoaldosteronism. J Clin Endocrinol Metab. 1995;80: 1929-1933.

20. Baker ME. Licorice and enzymes other than 11 beta-hydroxysteroid dehydrogenase: an evolutionary perspective. Steroids. 1994;59: 136-141.

21. Cristoni A, Magistretti MJ. Antiulcer and healing activity of Vaccinium myrtillus anthocyanosides. Farmaco Prat. 1987;42:29-43.

22. Magistretti MJ, Conti M, Cristoni A. Antiulcer activity of an anthocyanidin from Vaccinium myrtillus. Arzneimittelforschung. 1988;38 686-690.

23. Ogawa K, Oyagi A, Tanaka J, Kobayashi S, Hara H. The protective effect and action mechanism of Vaccinium myrtillus L. on gastric ulcer in mice. Phytother Res. 2011;25:1160-1165.

24. Huskisson EC, Jones J, Scott PJ. Application of visual analogue scales to the measurement of functional capacity. Rheumatol Rehabil. $1976 ; 15: 185-187$
Clinical and Experimental Gastroenterology

\section{Publish your work in this journal}

Clinical and Experimental Gastroenterology is an international, peerreviewed, open access journal, publishing all aspects of gastroenterology in the clinic and laboratory, including: Pathology, pathophysiology of gastrointestinal disease; Investigation and treatment of gastointes tinal disease; Pharmacology of drugs used in the alimentary tract;

\section{Dovepress}

Immunology/genetics/genomics related to gastrointestinal disease. This journal is indexed on CAS. The manuscript management system is completely online and includes a very quick and fair peer-review system. Visit http://www.dovepress.com/testimonials.php to read real quotes from published authors. 